\title{
ВОЛЬТАМПЕРОМЕТРИЧЕСКОЕ РАСПОЗНАВАНИЕ СОРТОВ МЕДА НА ОСНОВЕ МГК-АНАЛИЗА ВРЕМЕННЫХ РЯДОВ ТОКОВ
}

\author{
Максютова Э.И. ${ }^{1}$, Сидельников А.В. ${ }^{2}$ \\ ${ }_{1}^{1}$ ФБОУ ВО «Башкирский государственный университет», Уфа, Россия \\ ${ }^{2}$ ФГБОУ ВО Уфимский государственный нефтяной технический университет, Уфа, Россия \\ elzesha@gmail.com
}

DOI: 10.26902/ASFE-11_171

В последнее время большой интерес в аналитической химии вызывают мультисенсорные системы, позволяющие экспрессно идентифицировать природу многокомпонентных жидких и газовых смесей с использованием методов хемометрики. По аналогии с биологическими прототипами такие системы получили название «электронных языков», «электронных носов». Особенность мультисенсорных систем такого типа, с точки зрения практического применения, связана с возможностью обучать их распознаванию многокомпонентных смесей, минуя стадии качественного и количественного анализа исследуемого объекта. В данной работе предложены новые подходы к формированию многомерных аналитических сигналов для обучения вольтамперометрических систем типа «электронный язык» на основе временных рядов токов. Для сбора многомерной информации обычно применяются классические подходы, предусматривающие регистрацию массива данных объемом выборки по каждому сенсору порядка 10-30 значений. Это делается для оценки воспроизводимости с использованием нормального распределения.

В последние годы интерес представляют методы сбора информации предусматривающие большие объемы данных. Первоначально такие подходы (или как их называют Big Data) начали применять в макроэкономике, бизнесе и на предприятиях для извлечения скрытой информации, накапливаемой в течение продолжительного времени протекания тех или иных процессов. В концепции Big Data временной фактор является одним из определяющих при расшифровке внутренних/скрытых закономерностей в массивах данных. При составлении обучающей матрицы большие массивы данных формируются в виде временных рядов, объем которых достигает тысячи и миллионы значений. Токи, формируемые в условиях непрерывного функционирования вольтамперометрических сенсоров, представляют собой временные ряды, которые в рамках концепции Big Data использовали в работе для распознавания многокомпонентных растворов продукции пчеловодства.

В работе показано, что непрерывная регистрация вольтамперограмм (до нескольких сотен циклов регистрации вольтамперограмм) является необходимым условием для сборки аналитической информации и надежного распознавания близких по химическому составу сортов меда. При большом объеме выборки поверхность рабочего электрода под действием приложенного напряжения претерпевает многократные структурные изменения. В условиях протекания фарадеевских токов окисления/восстановления химических компонентов электрода и исследуемого раствора на границе раздела фаз в матрице временных рядов накапливается полезна аналитическая информация. В работе показано, что образцы меда различного происхождения формируют различную структуру (кластеры) во временных рядах токов в условиях непрерывной регистрации циклических вольтамперограмм.

В данной работе подобраны рабочие условия и определены аналитические характеристики сенсорных систем (чувствительность и специфичность) при вольтамперометрическом распознавании монофлерных и полифлерных образцов меда.

Работа выполнена при финансовой поддержке РФФИ, проект № 19-33-90191. 